\title{
Selective search in short-term memory under ideal conditions of test stimulus categorization
}

\author{
KLAUS-PETER MUTHIG and ROLF ULRICH \\ Psychologisches Institut der Universität Tübingen, Tübingen, Federal Republic of Germany
}

\begin{abstract}
It has often been claimed that short-term memory search is not selective because it takes longer to categorize the test stimulus than to search throughout the whole memory set. Four experiments investigated whether evidence for selective search can be obtained when effectively no time is required to categorize the test stimulus. Even under this favorable condition, no evidence was found for selective search of only the relevant memory subset. Therefore, the time to categorize a test stimulus cannot be the only reason why short-term memory search is not selective.
\end{abstract}

Search processes in short-term memory (STM) have been predominantly investigated within the itemrecognition paradigm (Sternberg, 1966, 1969). In this paradigm, a short list of sequentially displayed items (the memory list) has to be retained on every trial. After a brief delay, a single item (the test stimulus) is presented and a speeded yes/no decision is required, whether the test stimulus is a member of the memory list (positive trial) or not (negative trial). Reaction time (RT) is measured from the onset of the test stimulus to the occurrence of the response. Approximately linear increases of mean RT with memory list size for positive and negative trials have given strong evidence that the search through the items in STM (the memory set) is serial and exhaustive, and not self-terminating as common sense may suggest.

However, there is evidence that the search process changes if the memory lists contain items that belong to different categories: letters and digits, for instance (Jones \& Anderson, 1982; Lively \& Sanford, 1972; Naus, 1974; Naus, Glucksberg, \& Ornstein, 1972; Simpson, 1972; Ulrich \& Muthig, 1983). Two search models have become prominent in accounting for the altered search process. Both models assume that the memory set is partitioned according to the categories that make up the memory list (e.g., letter subset and digit subset). In addition, both models share the assumption that within each memory subset the search is serial and exhaustive. However, the two models differ with respect to how the subsets are entered in the search process.

\section{Directed-Entry Model}

According to this model, there are two stages of the search process: (1) the categorical membership of the test stimulus is determined; (2) the corresponding memory

We gratefully acknowledge the help of Markus Giray in computer programming and of Miles Hewstone in making this manuscript more readable. We also are indebted to Robert A. Bjork, Norman Slamecka, and one anonymous reviewer for their helpful comments on an earlier draft of this article. Requests for reprints should be sent to Klaus-Peter Muthig, Psychologisches Institut der Universität Tübingen, Friedrichstrasse 21, D-7400 Tübingen, Federal Republic of Germany. subset is searched serially and exhaustively. The categorical information of both the subset and the test stimulus is assumed to restrict the search to the relevant subset so that the speeded yes/no decision is made without searching the remaining irrelevant subsets in memory. Accordingly, the model predicts an equal increase of RT for positive and negative trials with increasing size of the relevant subset, but no increase of RT with increasing size of the irrelevant subset. If, for example, memory lists contain two equal-sized categorically distinct subsets of items, the RT functions for both positive and negative trials should be reduced by $50 \%$ compared with those resulting if homogeneous memory lists of equal size are used.

There is evidence from scanning studies using categorized memory lists that is in accordance with these predictions (e.g., Darley, Klatzky, \& Atkinson, 1972; Homa, 1973; Kaminsky \& DeRosa, 1972; Lively \& Sanford, 1972; Okada \& Burrows, 1973). However, the predictions of the directed-entry model are not always confirmed (e.g., Burrows \& Okada, 1973; Clifton \& Brewer, 1976; Crain \& DeRosa, 1974; Simpson, 1972). Strong evidence against the directed-entry model was provided by Simpson (1972, Experiment 2). In that study, positive and negative sets were drawn from different categories of stimuli (digits vs. letters, respectively). Hence, category membership information was sufficient and consistent to guide the speeded decision. However, the slopes of the RT functions obtained were not reduced compared with those for which negative probes from the same category were used (Simpson, 1972, Experiment 1).

That the available data do not give conclusive evidence of whether search is selective or not may depend on the respective relations between time savings (by only comparing a subset of items with the test stimulus) and additional time costs (by identifying the category of the test stimulus and locating the corresponding memory subset). If this is true, then a selective search strategy should be more probable, if the additional time costs can be reduced.

\section{Random-Entry Model}

In contrast to the directed-entry model, this model as- 
sumes a nondirected or random choice of the subsets to be searched. Within each subset, the comparison process is assumed to be serial and exhaustive, irrespective of a categorical correspondence between the subset searched and the test stimulus. Between subsets, the search process is assumed to be self-terminating: No other subset will be entered if a positive match has occurred in the presently searched subset. This random-entry strategy implies that on positive trials the relevant memory subset will always be searched, whereas irrelevant subsets are searched only in a fraction of trials (e.g., $50 \%$ in the case of only two subsets). Therefore, on positive trials, increasing the size of the relevant subset affects RT more than increasing the irrelevant subset by the same amount. However, on negative trials, all subsets have to be searched before a negative response can be given. If memory lists contain two equal-sized categorically distinct subsets of items, the slope of the RT function for positive trials should be reduced by $25 \%$ compared with the slope for negative trials.

Slope reductions very close to this prediction have been reported for memory lists containing words of equal-sized taxonomic categories (Naus, 1974; Naus et al., 1972), as well as for lists with letter/digit categorical distinction in which the two sublist sizes were also varied independently (Ulrich \& Muthig, 1983).

Although the available data suggest that both the directed-entry and the random-entry strategies can be followed in high-speed scanning of categorized lists, under which conditions the respective strategies are used remains a puzzle. Naus (1974) argued that the random-entry strategy may be preferred because membership determination of the test stimulus, necessary to direct search, is often too time consuming. Following a random-entry strategy, however, would still lead to faster RT than an exhaustive search of the memory set, due to the time savings on positive trials.

As in the case of the directed-entry model, this argument leads to the prediction that the directed-entry strategy should be more probable, the lower the time requirements of categorical membership determination of the test stimulus are (cf. Naus et al., 1972; Sternberg, 1975; Wescourt \& Atkinson, 1976). If this conjecture is true, then the directed-entry strategy should be followed with a probability close to one if effectively no time is required to determine the categorical membership of the test stimulus.

In the present study, four experiments were designed to test this prediction. In these experiments, the subjects were informed before each trial to which subset of list items the test stimulus belonged. The time required for membership determination of the test stimulus was hereby experimentally set to zero.

If no evidence for selective search can be obtained even under these favorable conditions, one must seriously doubt the view that selective memory search is hindered because membership determination of the test stimulus is too time consuming. Indeed, that information about the membership of the test stimulus may be immediately available is suggested by visual search (e.g., Jonides \& Gleitman, 1972, 1976) and partial report (e.g., Duncan, 1983) studies. If this is true for memory search, too, other conceptualizations are needed to explain why memory search is not selective.

\section{GENERAL METHOD}

Because the four experiments were very similar in method, we present a General Method section before providing the rationale and results for each experiment.

\section{Subjects}

The subjects were introductory psychology students who received partial fulfillment of a curriculum requirement. There were 35,4 , 36, and 40 subjects in Experiments 1-4, respectively.

\section{Paradigm}

An extension of Sternberg's (1969) varied-set procedure was used. There were two different types of trials: additional set trials and control trials. In additional set trials (see Figure 1), memory lists of size $S$ were composed of two equal-sized subsets, the target set $\mathrm{X}(1), \ldots, \mathrm{X}(\mathrm{S} / 2)$ and the additional set $\mathrm{Y}(1), \ldots, \mathrm{Y}(\mathrm{S} / 2)$, respectively. In Experiments 1-2, the members of the target and the additional set were from different categories (letters and digits); in Experiments 3-4, the target set and additional set items belonged to the same category (digits). The items of the two subsets were presented differently on the screen: bright items against a dark background (target set) and dark items against a bright background (additional set). The subjects were instructed that the test stimulus could be a member of only target set $X$ (on positive trials) or be neither from the target set $X$ nor from the additional set $Y$, but from $Z$, the complement of both subsets (on negative trials). Therefore, in order to give a correct response, it was not necessary to search the additional set. To ensure that the subjects also committed the additional set to memory, in $50 \%$ of the trials the additional set items had to be recalled after the yes/no decision.

In control trials, all items of the memory list were relevant for the yes/no decision. Accordingly, target set and additional set items were not presented differently on the screen. Test stimuli could be members of $\mathrm{X}, \mathrm{Y}$, or $\mathrm{Z}$ on these trials.

\section{Procedure}

The subjects were required to memorize lists of 2,4 , or 6 sequentially presented items on each trial, with all of the items from one subset appearing before any of the items from the other subset. The sequence of the two subsets was randomized across trials, with the restriction that both subsets appear equally often in the first and in the second position of the input string. After the last memory list item had been displayed, an end-of-list marker appeared on the screen. Thereafter, the test stimulus was displayed until the subjects had responded. The subjects were required to make a speeded yes/no decision whether the test stimulus was a member

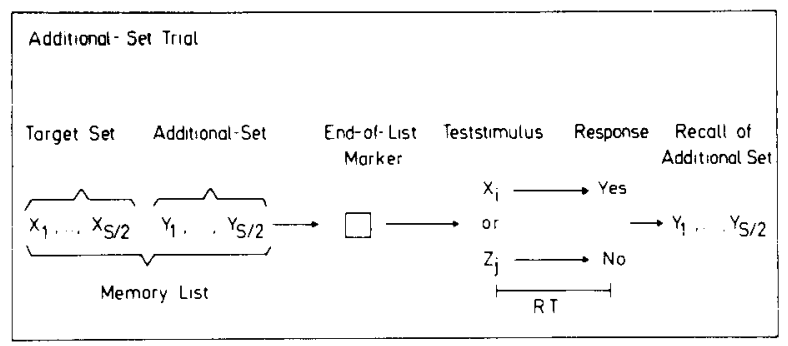

Figure 1. A prototype additional set trial for memory lists of size $S$. 
of the target set or not. In $50 \%$ of the additional set trials, free recall of the additional set items was required afterwards.

At the beginning of each trial, a fixation dot marked the center of the viewing area and served as a cue to start the next trial. Pushing the initiation button replaced the fixation dot with a visual cue (Experiments 1-3) that indicated the type of trial (additional set trial vs. control trial). Thereafter, the memory list items were presented sequentially for $900 \mathrm{msec}$ each, with an interstimulus interval of $300 \mathrm{msec}$. Nine hundred milliseconds after the last memory list item, an end-of-list marker was displayed for $900 \mathrm{msec}$. One second later, the test stimulus was displayed until the subjects had responded.

The subjects were encouraged to respond quickly and to keep error rates low. Errors were fed back by an "ERROR" cue whose flickering time was proportional to the error rate made up to the present trial. RTs longer than $1.5 \mathrm{sec}$ were reported back by displaying "FASTER RESPONDING REQUIRED" on the screen.

After the subjects had responded to the test stimulus in the additional set condition, "RECALL ADDITIONAL SET" was displayed with probability .5 on the screen. Recall of the additional set items was required via the keyboard of the computer. Error feedback was given to any false recalled item.

\section{Memory List Items}

Memory lists of sizes $S$ ( $S=2,4,6)$ were constructed of letters A-I and digits $1-9$, using standard CBM 3032 characters (matrix of 7 $\times 6$ dots). Legibility and discriminability of these characters are sufficiently high to prevent systematic confusion errors between any pair of letters and digits used. Letters and digits were randomly assigned to memory lists and were not repeated within a memory list

\section{Design}

Twelve conditions were produced by a 2 (response type: positive vs. negative) $\times 2$ (trial type: additional vs. control) $\times 3$ (list size: $2,4,6$ ) factorial design. In Experiments 1-3, all experimental manipulations were within subjects. In Experiment 4, trial type was made a between-subjects factor: The subjects were run either in the additional set condition or in the control set condition. Every subject completed 60 training trials and 120 experimental trials in one 45-min session (Experiments 1, 3, and 4). The four subjects in Experiment 2 completed 1,200 experimental trials in 10 successive sessions of 30 min each day.

\section{Equipment}

The experiments were controlled by a CBM 3032 personal computer. Memory list items and test stimuli were presented on the screen. Viewing distance was approximately $70 \mathrm{~cm}$. On a separate device, there were three response buttons arranged in a triangle. With the two upper buttons, the subjects had to indicate their yes/no decisions. The lower one served as trial-initiation button. Positions of yes and no buttons were counterbalanced across subjects.

\section{EXPERIMENT 1}

\section{Rationale}

The purpose of Experiment 1 was to investigate whether selective search of categorized lists is enhanced when actually no time is required to determine the categorical membership of the test stimulus.

\section{Results and Discussion}

RTs were discarded for trials in which an error was made or the time itself exceeded $1.5 \mathrm{sec}$ (less than $1 \%$ of the correct responses). Recognition error rates across conditions ranged from $0 \%$ to $5 \%$, averaging $2.5 \%$. There was no indication of a speed-accuracy trade-off in the data. Recall error rates across conditions ranged from $8 \%$ to
$19 \%$. On positive trials, they slightly increased with list size; on negative trials, no such increase was observed. However, generally more errors were made on negative trials $(17.3 \%$, on the average) than on positive trials $(10.7 \%)$

The relationship between RT performance and list size for each combination of trial type and response type is depicted in Figure 2. Each point on the graph is the average of approximately 342 trials. Standard errors of the means ranged from 14 to $20 \mathrm{msec}$, slightly increasing with list size, and averaged $16.4 \mathrm{msec}$.

RTs were submitted to an analysis of variance. RTs were significantly longer for no than for yes decisions $[\mathrm{F}(1,3780)=38.8, \mathrm{p}<.001]$ and increased with list size $[F(2,3780)=128.6, p<.001]$. Although the overall RT for control sets was $10 \mathrm{msec}$ longer than that for additional sets, the respective main effect did not reach significance $[F(1,3780)=2.0, p>.10]$.

Mean RTs show linear list size effects for both the additional set and the control set condition. Linear regression accounted for $99 \%$ of the variance in the additional set condition and $96 \%$ in the control set condition. The only condition in which the data are not very closely fit by a linear function is the positive control. The reason for this departure from linearity remains unclear. At least there is no indication that this comes about because of unusually large variance in the respective cells.

Within each condition, positive and negative slopes can be regarded as parallel $[\mathrm{F}(2,3780)=1.2, \mathrm{p}>.25]$. Be-

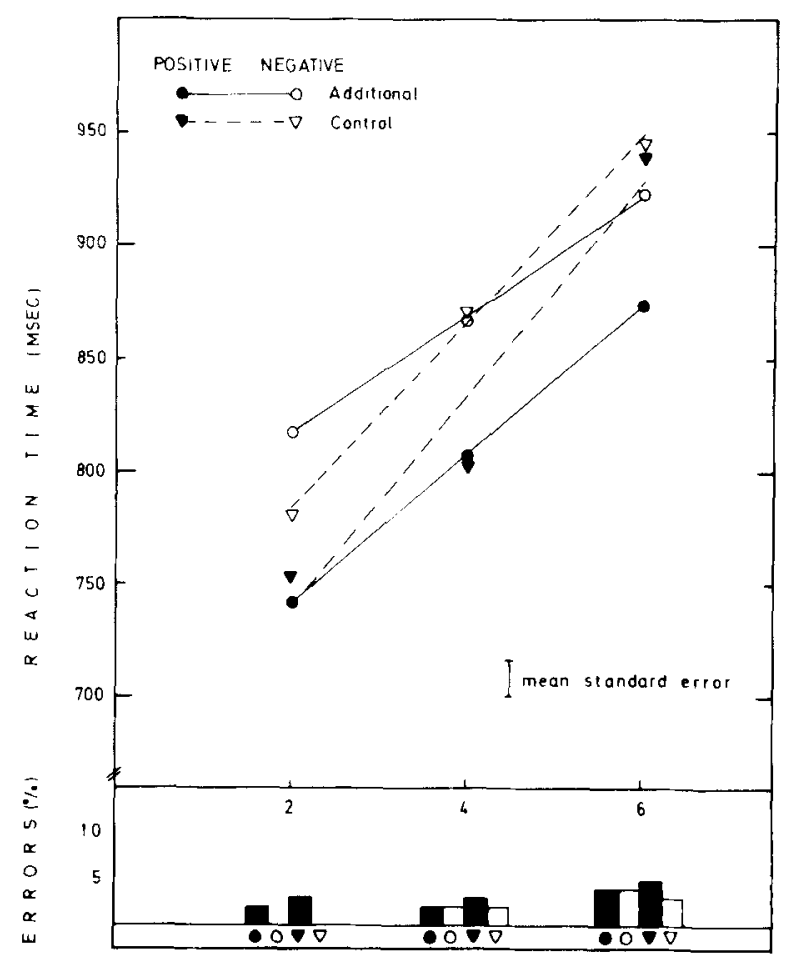

Figure 2. Data from Experiment 1: Mean RTs for correct responses and percentage of recognition errors as a function of list size. 
tween conditions, slopes differ significantly $[\mathrm{F}(2,3780)$ $=5.2, \mathrm{p}<.01$ ] (33 and $26 \mathrm{msec}$ in the additional set condition vs. 47 and $41 \mathrm{msec}$ in the control condition). This corresponds to a slope reduction of $30 \%$ for positive trials and $37 \%$ for negative trials in the additional set condition compared with the control condition. However, the intercepts of the RT functions for both positive and negative trials were greater in the additional set condition than in the control condition (676 and $765 \mathrm{msec}$ vs. 643 and $702 \mathrm{msec}$, respectively). The intercept difference between positive and negative RT functions was smaller in the control condition than in the additional set condition, yet did not reach significance $[\mathrm{F}(1,3780)=3.5, \mathrm{p}<.10]$.

The obtained parallel slopes of positive and negative RT functions within each condition are compatible with the directed-entry model but incompatible with the random-entry model. However, the obtained slope reductions are far from what can be expected (50\% reduction) if memory search is restricted to the relevant subset only. Hence, although the subjects were informed about the relevant subset in advance, there is no evidence that they did follow a pure directed-entry strategy. Instead, the present data seem to indicate that a mixture of two strategies was used in additional set trials: In some proportion of trials, both target and additional set were searched, whereas in the other, only the target set was searched. Introspective reports of the subjects also support this conjecture. In postexperimental interviews, many subjects reported that for additional set trials they often had difficulties in rehearsing the items of the two subsets separately. Thus, selective chunking of the items of the two subsets was tried but probably was not always possible.

\section{EXPERIMENT 2}

\section{Rationale}

The lack of evidence for a pure directed-entry strategy in Experiment 1 might be due to difficulties in selective chunking by the (untrained) subjects. Because selective chunking should be easier to obtain with prolonged training, selective search also should be more probable for trained subjects. If this is true, then prolonged training should lead to a more profound reduction of slopes $(\mathrm{m})$ in the additional set condition (A) than in the control set condition (C). Accordingly, the slope ratio, $m(A) / m(C)$, of the respective RT functions should decrease with practice. Therefore, Experiment 2 was designed as a replication of Experiment 1, with the exception that four subjects were tested on 10 successive sessions of 120 trials each. Also, the subjects were advised that selective chunking of the categorical distinct subsets might lead to better performance.

\section{Results and Discussion}

RTs were analyzed as in Experiment 1. The pattern of error rates was generally similar to that obtained in Experiment 1 , with the exception that this time recall errors slightly decreased with list size on positive trials. The overall error rate in item recognition was $1.7 \%$. Error rates in recalling the items of the additional set were considerably smaller in Experiment 2, ranging from $4.3 \%$ to $8.1 \%$, averaging $5.8 \%$ on positive trials and $6.8 \%$ on negative trials.

Mean RTs for each combination of response type, trial type, and list size are displayed in Figure 3. Each point in Figure 3 is based on approximately 393 observations. Standard errors of the means ranged from 7 to $12 \mathrm{msec}$, and averaged $9.6 \mathrm{msec}$.

As in Experiment 1, RT was longer for no than for yes decisions $[F(1,4752)=110.5, p<.001]$ and increased with list size $[\mathrm{F}(2,4752)=233.6, \mathrm{p}<.001]$. The overall RT for control trials was slightly but not significantly longer than that for additional set trials $[F(1,4752)=1.0]$.

Again, the RT data show marked linear list size effects for both trial types. Linear regression accounted for $99 \%$ of the variance in both conditions.

In each condition, the slopes of the RT functions can be regarded as parallel $[F(2,4752)=0,10]$. The slopes in the control set condition were exactly the same for positive and negative trials ( $39 \mathrm{msec}$ each). These slopes are smaller than those in Experiment 1 but agree fairly well with the values reported by studies using homogeneous memory lists. In the additional set condition, slopes of $28 \mathrm{msec}$ were obtained for positive trials, and of $30 \mathrm{msec}$ for negative trials. Again this slope reduction (about $25 \%$ ) is significant $[\mathrm{F}(2,4752)=5.1, \mathrm{p}<.01]$, although smaller

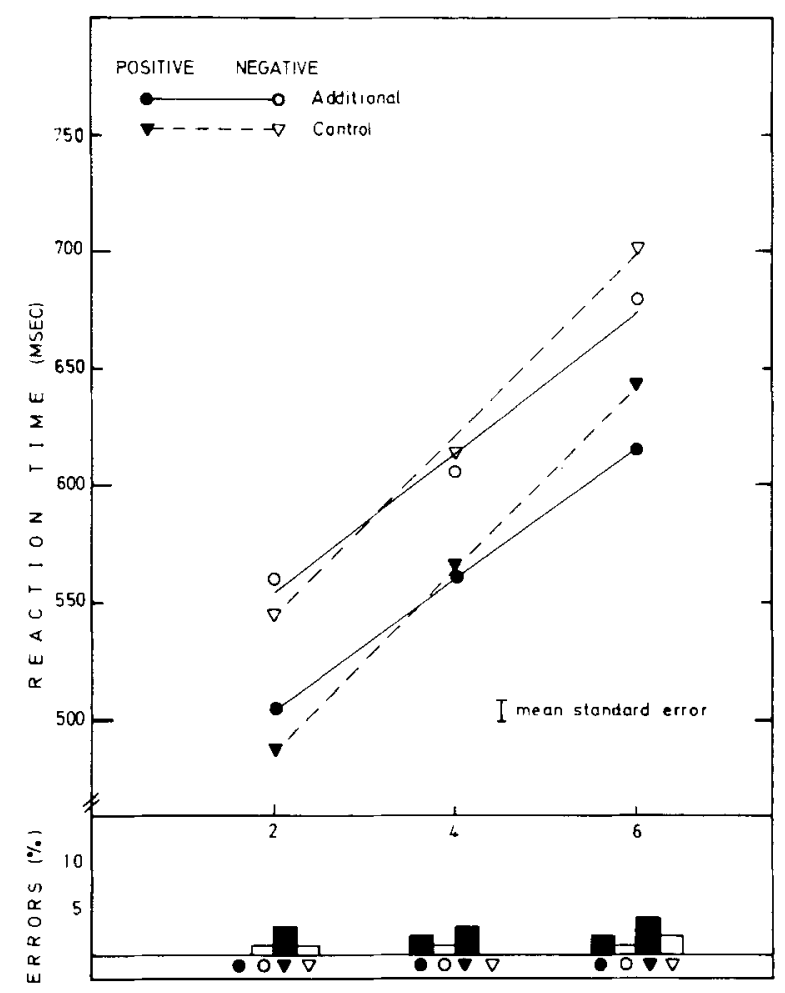

Figure 3. Data from Experiment 2: Mean RTs for correct responses and percentage of recognition errors as a function of list size. 
Table 1

Slopes (in Milliseconds) of the Individual RT Functions for Additional Set Trials and Control Trials in Sessions 1-5 and 6-10

\begin{tabular}{cccc}
\hline Subject & $\begin{array}{c}\text { Additional } \\
\text { Set Trials }\end{array}$ & $\begin{array}{c}\text { Control } \\
\text { Trials }\end{array}$ & $\mathrm{m}(\mathrm{A}) / \mathrm{m}(\mathrm{C})$ \\
\hline & \multicolumn{3}{c}{ Sessions $1-5$} \\
1 & 28 & 40 & \\
2 & 39 & 43 & 0.70 \\
3 & 37 & 36 & 0.91 \\
4 & 32 & 39 & 1.03 \\
& & Sessions 6-10 & 0.82 \\
1 & 18 & 28 & 0.64 \\
2 & 28 & 38 & 0.74 \\
3 & 20 & 38 & 0.53 \\
4 & 30 & 43 & 0.70 \\
\hline
\end{tabular}

than in Experiment 1 (about 33\%). As in Experiment 1, the intercepts of the RT functions are higher in the additional set condition (448 and $494 \mathrm{msec}$ for positive and negative trials, respectively) than in the control condition (408 and $465 \mathrm{msec}$ ). In contrast to Experiment 1, there was no indication of a response type $\times$ trial type interaction $[F(1,4752)=0.01]$.

The pattern of results obtained in Experiment 2 is fairly consistent with the results and interpretation of Experiment 1 . Again, a random-entry strategy of memory search can be rejected on the basis of the data, because there is no indication of nonparallel RT functions in both set conditions. Yet, there is again no evidence for a pure selective search in the additional set condition.

\section{Practice Effects}

To test whether there is more evidence for selective memory search with practice, the respective slopes of Sessions 1-5 and 6-10 were computed for each subject and submitted to a response type $\times$ trial type $\times$ session block $x$ subject ANOVA.

Results revealed a significant slope reduction (from 37 to $31 \mathrm{msec})$ due to practice $[\mathrm{F}(1,128)=4.3, \mathrm{p}<.05]$, which is the same for positive and negative trials $[\mathrm{F}(1,128)$ $=0.02]$. As expected, there is a tendency for a more profound slope reduction in the additional set condition $[\mathrm{F}(1,128)=2.8, \mathrm{p}<.10]$. The slope ratio $\mathrm{m}(\mathrm{A}) / \mathrm{m}(\mathrm{C})$ decreased with practice from 0.87 to 0.65 (see Table 1 ). This is in accord with the assumption that practice improves selective chunking and therefore makes selective search more likely.

\section{EXPERIMENT 3}

\section{Rationale}

The slope reductions obtained in Experiments 1-2 are far from the $50 \%$ to be expected if subjects use a pure directed-entry strategy in the additional set condition. One might argue that this is because in some cases memory sets were also searched selectively in the control condition. Although in the control condition the subjects were not informed of the target set in advance, a selective search strategy could have been used via categorization of the test stimulus before the target set was scanned. In such a case, the slope ratio $\mathrm{m}(\mathrm{A}) / \mathrm{m}(\mathrm{C})$ is not decisive as to whether a pure selective search strategy is used in the additional set condition. Assume, for example, that the target set is always selectively searched in the additional set condition and with probability .5 in the control one. It can be shown that for this case a slope reduction of $33 \%$ must result. ${ }^{1}$ Therefore, Experiments 1-2 are not completely conclusive as to whether a pure directed-entry strategy is used in the additional set condition.

Only if the possibility of categorizing the test stimulus in the control set condition can be ruled out do the resulting slope reductions directly reflect the extent to which a direct-entry strategy is used.

To prevent categorization of the test stimulus in the control condition, homogeneous memory lists (digits only) were used in Experiment 3. With respect to all other conditions, Experiment 3 was a replication of Experiment 1.

\section{Results and Discussion}

RTs were analyzed as in the two preceding experiments. Recognition error rates across conditions ranged from $0 \%$ to $6 \%$. The overall item-recognition error rate was $2.4 \%$. Recall errors averaged $14.2 \%$. Again, they increased with list size on positive trials but were relatively unaffected by list size on negative trials. As in the preceding experiments, the mean recall error rate was higher on negative trials $(16.3 \%)$ than on positive trials $(12 \%)$. With respect to error rates, Experiment 3 is almost identical to Experiment 1.

Mean RTs for Experiment 3 are displayed in Figure 4. Each point on the graph is the mean of about 351 trials. Standard errors of the means ranged from 10 to $22 \mathrm{msec}$, and averaged $15.4 \mathrm{msec}$. Again, the RT data show linear list size effects for both conditions. This time, linear regression accounted for $98 \%$ of the variance in the additional set condition and $99 \%$ of the variance in the control set condition.

Analysis of variance revealed a significant main effect of list size $[F(2,3888)=164.5, p<.001]$ and three significant interactions: (1) The mean RT difference between positive and negative trials was smaller in the additional set condition $(13 \mathrm{msec})$ than in the control condition $(39 \mathrm{msec})[\mathrm{F}(1,3888)=14.7, \mathrm{p}<.01]$. (2) The $\mathrm{RT}$ difference between positive and negative trials diminished with list size in both conditions from about 40 to $20 \mathrm{msec}$, due to slightly steeper RT functions for positive trials $[F(2,3888)=6.8, p<.01]$. (3) As in the preceding experiments, slopes of the RT functions for positive and negative trials were significantly reduced in the additional set condition ( 39 and $36 \mathrm{msec}$ ) compared with the control $(55$ and $49 \mathrm{msec})[\mathrm{F}(2,3888)=11.0, \mathrm{p}<.001]$. This corresponds to a slope reduction of $29 \%$ and $27 \%$ for positive and negative trials, respectively.

The pattern of results obtained is fairly consistent with those obtained in the preceding experiments. Again, there is evidence that memory search under the control set con- 


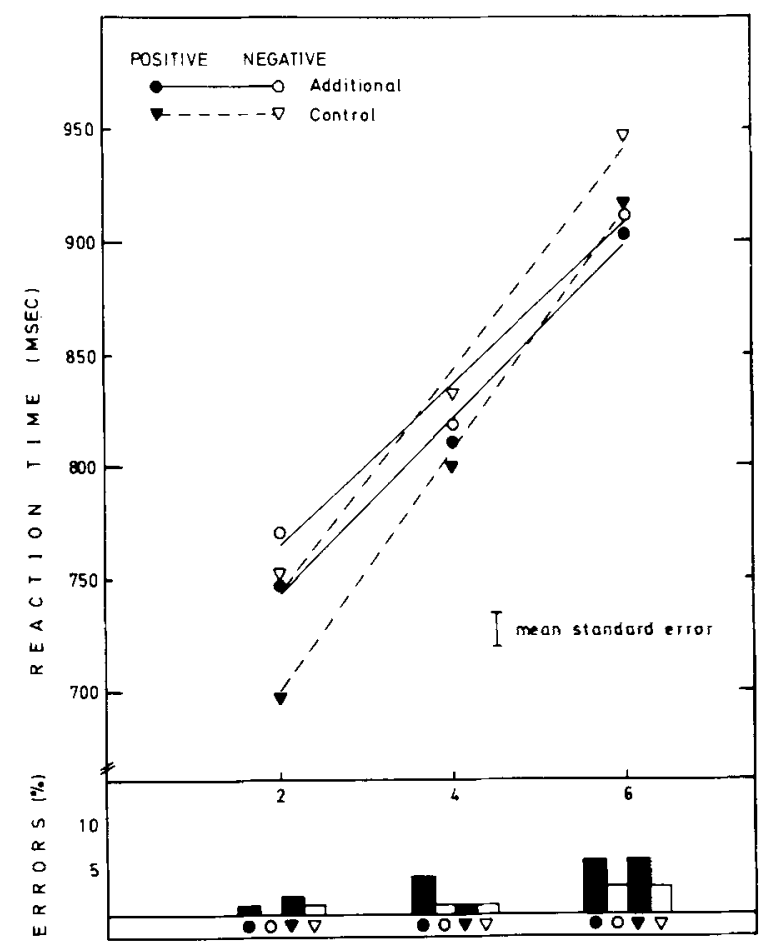

Figure 4. Data from Experiment 3: Mean RTs for correct responses and percentage of recognition errors as a function of list size.

dition is serial and exhaustive, whereas under the additional set condition a mixture of a directed-entry strategy and a pure exhaustive strategy is used. Since in this experiment the homogeneous memory lists did not allow for a selective search in the control condition, too, the slope reduction obtained directly indicates that in only $36 \%$ of the additional set trials was a selective search strategy used. ${ }^{1}$

\section{EXPERIMENT 4}

\section{Rationale}

It might be objected that the random intermixture of additional set and control set trials prevents subjects from establishing a pure directed-entry strategy, because they have to be ready to shift strategies (selective vs. unselective) unpredictably across trials. This shifting may have discouraged the consistent application of a selective search strategy in the additional set trials of Experiments 1-3. The lack of evidence for selective search therefore might be due to the within-subject treatment of trial type in these experiments rather than to a general difficulty in carrying out selective search.

If this objection is true, then a between-subjects treatment of trial type should yield more favorable conditions for subjects to exhibit a pure directed-entry strategy because under these conditions a consistent application of selective search is encouraged.

Experiment 4 was designed to test whether stronger evidence for a pure selective search can be found in addi- tional set trials, if trial type is made a between-subjects factor. Except for this change in design, Experiment 4 was a replication of Experiment 3.

\section{Results and Discussion}

RTs were analyzed as in the preceding experiments. Generally, fewer errors were made this time, but the pattern of errors was nearly the same as that in Experiment 3. Recognition errors averaged $1.9 \%$. Recall errors ranged from $2 \%$ to $9 \%$, and averaged $2.7 \%$ for positive trials (again slightly increasing with list size) and $7.7 \%$ for negative trials (again not increasing with list size).

Mean RTs for each combination of response type, trial type, and list size are displayed in Figure 5. Each mean is based on approximately 374 observations. Standard errors of the means were considerably smaller this time, ranging from 9 to $14 \mathrm{msec}$ and averaging $10.9 \mathrm{msec}$.

Again, the RT data show marked linear list size effects for both trial types. As in Experiment 3, 98\% of the variance in the additional set condition and $99 \%$ of the variance in the control set condition were accounted for by linear regression.

Analysis of variance revealed two significant main effects: (1) RTs were longer on negative than on positive trials $[F(1,38)=20.27, \mathrm{p}<.001]$, and (2) RTs increased with list size $[F(2,76)=181.64, p<.001]$. Qualitatively, the data show a pattern of interactions similar to that in Experiment 3. That these interactions did not reach statistical significance this time might be due to the

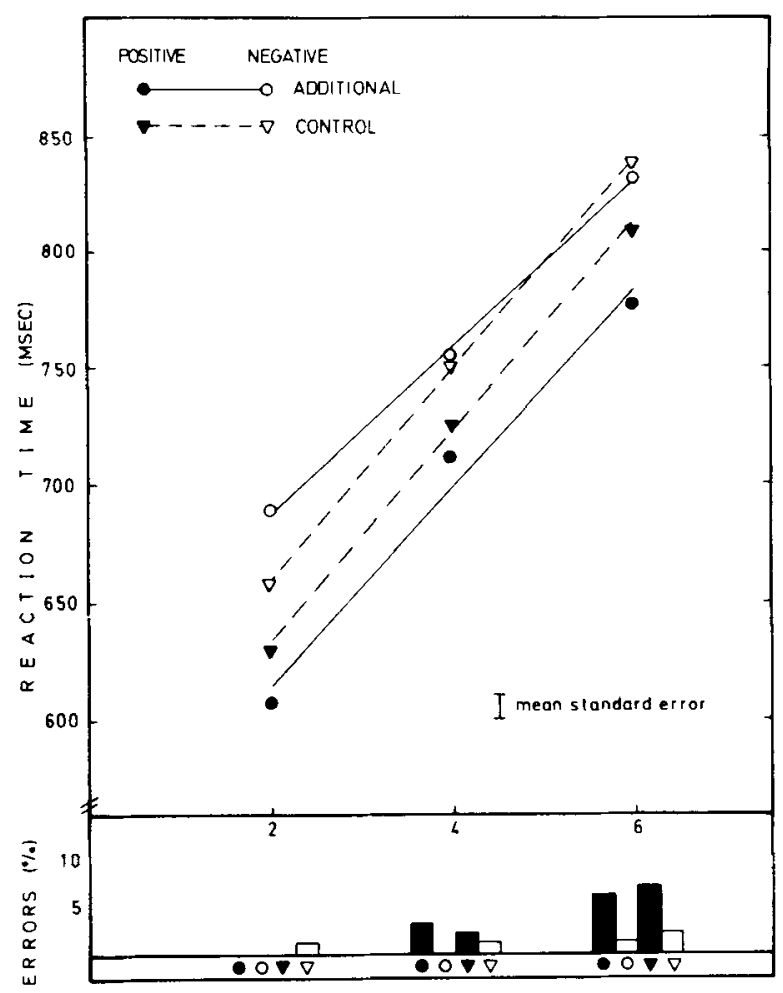

Figure 5. Data from Experiment 4: Mean RTs for correct responses and percentage of recognition errors as a function of list size. 
reduced sensitivity of the between-subjects design compared with the within-subject design of the preceding experiment: Mean RT differences between positive and negative trials tended to be smaller in the control set condition $(28 \mathrm{msec})$ than in the additional set condition $(59 \mathrm{msec})[\mathrm{F}(1,38)=3.41, .05<\mathrm{p}<.10]$. In Experiment 3 , however, this difference was found to be smaller for additional set trials. As in Experiment 3, the RT difference between positive and negative trials diminished with list size from about 55 to $41 \mathrm{msec}[\mathrm{F}(2,76)=1.41, .10$ $<\mathrm{p}<.25$ ].

The most remarkable feature of the present data is that this time slopes of the RT functions for positive and negative trials are only slightly reduced in the additional set condition (42 and $35 \mathrm{msec}$ ) compared with the control (45 msec for both) $[F(2,76)=.64]$. Taking into account that the between-subjects treatment of trial type was expected to encourage the consistent application of a directed-entry strategy, this is a surprising result. Since postexperimental interviews of the subjects run in the additional set condition revealed that most of them tried one or another strategy of chunking and rehearsing the two subsets separately, one can only speculate why the present result differs from the results obtained in Experiments 1, 2 , and 3. Probably, the application and refinement of a directed-entry strategy is critically dependent on the presence of contrasting conditions with which its efficiency can be compared.

However, the main point that can be made of this result is that again there is no evidence in favor of the notion that search in STM is completely selective. Although this time the consistent application of a directed-entry strategy was encouraged, the between-subjects design obviously did not make the execution of a directed-entry search more probable than the formerly used within-subject design. Accordingly, the results obtained in Experiments 1-3 are hardly attributable to the random intermixture of control set and additional set trials in these experiments.

\section{GENERAL DISCUSSION}

The present results show that the scanning process in STM is altered if the subset relevant for search is known in advance. However, although there is evidence that a directed-entry strategy can be followed under this condition, there is no evidence that a pure directed-entry strategy is followed consistently. The mean slope reductions in the present experiments are all clearly less than the $50 \%$ reduction to be expected if memory search is completely selective. Instead, the present results indicate that, at best, a mixture of a directed-entry and an exhaustive search strategy is used, even when there is valid information about the relevant memory subset in advance, and even when a consistent application of a directed-entry strategy is encouraged.

This is in contrast to studies in which data in favor of a pure directed-entry strategy are reported (e.g., Darley et al., 1972; Kaminsky \& DeRosa, 1972). If the proba- bility of following a directed-entry strategy is computed from the slope reductions reported in these earlier studies, probabilities of .93 and 1.11 (!) result, indicating that memory search was in fact highly selective in these experiments. However, there are differences with respect to the present study that may be responsible for the conflicting evidence.

In the study reported by Darley et al. (1972), the target set was always of size $S=1$, whereas in the present study, half of the memory set elements always constituted the target set. This raises the possibility that memory search is completely selective only if the target set does not contain more than one element. There are at least two arguments that support this conjecture: (1) In order to restrict memory search to the target set only, the memory set must be partitioned. However, a partitioning is probably easier if only one element has to be made more salient in contrast to all others (von Restorff, 1933). (2) A buffer model analogous to the expectancy model of Shiffrin and Schneider (1974) can be considered. Assume that in a matching buffer the test stimulus is compared with the elements of the memory set that are loaded serially into the matching buffer, regardless of the occurrence of a positive match. For partitioned memory sets, the elements of the target set are loaded with a higher probability than the elements of the additional set. If the buffer is loaded before the test stimulus is presented, then RT does not depend on the size of the additional set if the target set is of size $S=1$, as reported by Darley et al., (1972). Whenever the target set is of size S $>1$, RT depends on the size of the additional set, because its elements are also loaded in the matching buffer with a probability different from zero, as in the present study.

Discrepancies between the present results and those reported by Kaminsky and DeRosa (1972) may be due to two essential experimental differences between the studies: (1) Kaminsky and DeRosa did inform their subjects of the memory subset relevant for search, not before, but after, the memory list had been presented (the cue appeared $2 \mathrm{sec}$ before the test stimulus). (2) Even more important is the fact that no recall of the additional set items was required after completion of the search task. This allowed for deletion of the additional set before starting the search process. The results reported by Kaminsky and DeRosa (1972) are therefore not very informative as to whether memory search can be purely selective.

\section{CONCLUSION}

Two assumptions are usually made to explain the possibility of selective memory search: (1) The memory set has to be partitioned, and (2) information about the categorical membership of the test stimulus must be gained to guide the search.

That memory sets can be partitioned, at least when categorized memory lists are used, usually seems to be evident and unquestioned. Whether memory search is selective or not is therefore attributed to the categoriza- 
tion of the test stimulus, or, more precisely, to the time it takes to categorize the test stimulus and to locate the relevant subset in memory. In the experiments reported here, the (categorical) membership information about the test stimulus was provided in advance, so that the subset relevant for search could also have been located in advance. Because no additional time is required to categorize the test stimulus, the advantage of a restricted search according to a directed-entry strategy should pay off fully. However, evidence for a pure directed-entry strategy was definitely not obtained in the present experiments. Consequently, if one agrees that providing information about the relevant subset in advance reduces the time required to categorize the test stimulus to zero, then the lack of evidence for selective search can no longer be attributed to a time-consuming categorization process.

Although the present results give evidence for an altered scanning process if the relevant subset is known in advance, the kind of alteration obtained can be given no plausible interpretation within the framework of a directed-entry model or a random-entry model.

Nevertheless, these models have had their value as first conceptualizations of selective search. However, alternative conceptualizations of selective search in STM are needed in order to account for the present findings. A first step in this direction would be to get a precise answer to the question of whether and how irrelevant information simultaneously present in STM is involved in any comparison process. The present data suggest that answers to these questions may be found more easily if one is not confined to the specific assumptions of the directed-entry model.

\section{REFERENCES}

Burrows, D. \& OKada, R. (1973). Parallel scanning of semantic and formal information. Joumal of Experimental Psychology, 97, 254-257.

Clifton, C., \& BREWER, E. (1976). Partially selective search of memory for letters and digits. Memory \& Cognition, 4, 616-626.

Crain, R. D., \& DeRosa, D. V. (1974). Retrieval of information from multiple ensembles in short-term memory. Memory \& Cognition, 2, 255-260.

Darley, C. F., Klatzky, R. L., \& Atkinson, R. C. (1972). Effects of memory load on reaction time. Journal of Experimental Psychology, 96, 232-234.

DunCaN, J. (1983). Perceptual selection based on alphanumeric class: Evidence from partial reports. Perception \& Psychophysics, 33, 533-547.

HomA, D. (1973). Organization and long-term memory search. Memory \& Cognition, 1, 369-379.

JONES, W. P. . \& ANDERSON, J. R. (1982). Semantic categorization and high-speed scanning. Joumal of Experimental Psychology: Leaming, Memory, and Cognition, 8, 237-242.

Jonides, J., \& Gleitman, H. (1972). A conceptual category effect in visual search: $\mathrm{O}$ as letter or as digit. Perception \& Psychophysics, $12,457-460$.

Jonides, J., \& Gleitman, H. (1976). The benefit of categorization in visual search: Target location without identification. Perception \& Psychophysics, 20, 289-298.

Kaminsky, C. A., \& DeRosA, D. V. (1972). Influence of retrieval cues and set organization on short-term recognition memory. Journal of Experimental Psychology, 96, 449-454.

Lively, B. L., \& SANFord, B. J. (1972). The use of category information in a memory-search task. Journal of Experimental Psychology, 93, 379-385.

NAUS, M. J. (1974). Memory search of categorized lists: A consideration of alternative self-terminating search strategies. Journal of Experimental Psychology, 102, 992-1000.

Naus, M. J., Glucksberg, S., \& Ornstein, P. A. (1972). Taxonomic word categories and memory scarch. Cognitive Psychology. 3, 643-654

OKaDA, R., \& Burrows, D. (1973). Organizational factors in highspeed scanning. Journal of Experimental Psychology, 101, 77-81.

SHIFFrin, R. M., \& SCHNEIDER, W. (1974). An expectancy model for memory search. Memory \& Cognition, 2, 616-628.

Simpson, P. J. (1972). High-speed memory scanning: Stability and generality. Journal of Experimental Psychology, 96, 239-246.

SternBerg, S. (1966). High-speed scanning in human memory. Science, $153,652-654$.

SternberG, S. (1969). Memory scanning: Mental processes revealed by reaction-time experiments. American Scientist, 57, 421-457.

SternberG, S. (1975). Memory scanning: New findings and current controversies. Quarterly Journal of Experimental Psychology, 27, 1-32.

UlRICH, R., \& MUthIG, K. P. (1983). Darbietungsabhängige Vergleichsprozesse bei kategorial zerlegbaren Gedächtnismengen. Zeitschrif für experimentelle und angewandte Psychologie, 30, 673-694.

vON RESTORFF, H. (1933). Über die Wirkung von Bereichsbildungen im Spurenfeld. Psychologische Forschung, 18, 299-342.

Wescourt, K. T., \& AtKInson, R. C. (1976). Fact retrieval processes in human memory. In W. K. Estes (Ed.), Handbook of learning and cognitive processes (Vol. 4, pp. 363-413). Hillsdale, NJ: Erlbaum.

\section{NOTE}

1. Relation between the observed slope reduction $[m(A) / m(c)]$ and the probability $(p)$ of using a directed-entry strategy in the additional set condition-Assume that with probability p only the target set is searched in the additional set condition. In this case, the expected RT for list size $\mathrm{S}=\mathrm{s}$ is: $\mathrm{E}(\mathrm{RT} / \mathrm{s}$, directed $)=\mathrm{m} * \mathrm{~s} / 2+\mathrm{c}$. If instead the whole memory set is searched, the expected RT is: $E(R T / s$, nondirected $)=m^{*} s+c$. Since the observed RT is assumed to be a mixture of directed and nondirected search, we have: $E(R T / s)=p\left(m^{*} s / 2+c\right)+(1-p)^{*}\left(m^{*} s+c\right)$. Simplification yields $E(R T / s)=(1-p / 2)^{*} m^{*} s+c$. Assuming that in the control set condition $p=0$, the slope ratio $m(A) / m(C)$ equals $(1-p / 2)$, from which the probability $p=2 *(1-m(A) / m(C))$ follows.

(Manuscript received July 23, 1984; revision accepted for publication August 28, 1984.) 